\title{
Overlapping Control Design for Multi-Channel Systems
}

\author{
Javad Lavaei ${ }^{\text {a }}$, Amir G. Aghdam ${ }^{\text {b }}$ \\ ${ }^{a}$ Department of Control and Dynamical Systems, California Institute of Technology, Pasadena, CA 91125, USA \\ ${ }^{\mathrm{b}}$ Department of Electrical and Computer Engineering, Concordia University, Montréal, QC H3G 1M8, Canada
}

\begin{abstract}
This paper deals with the decentralized overlapping control of interconnected systems. The notion of a quotient overlapping fixed mode (QOFM) is first introduced and it is shown that a mode of an interconnected linear time-invariant system can be shifted by means of a general decentralized overlapping controller if and only if it is not a QOFM. It is then asserted that any interconnected system with no unstable QOFM can be stabilized by using an appropriate finite-dimensional linear timevarying controller. It is also shown how the existing results aiming at designing a decentralized controller of a certain type such as generalized sampled-data hold function, finite-dimensional linear time-varying, and sampled-data can be utilized to design a decentralized overlapping controller of a desired form, in order to achieve any design specifications. The efficacy of the results is elucidated through two numerical examples.
\end{abstract}

Key words: Decentralized control, Graph theory, Time-varying control

\section{Introduction}

Control of interconnected systems has been of great interest in the literature in the past three decades, due to its wide range of applications in important real-world problems. Such applications include power systems, communication networks, flexible space structures, etc. to name only a few. Due to the distributed nature of the problems of this type, the conventional control techniques are often not capable of handling them efficiently. More specifically, it is desired in the distributed interconnected systems to impose some constraints on the structure of the controller to be designed. These constraints determine the outputs of which subsystems could contribute to the construction of the input of each subsystem. To formulate the control problem, these constraints are usually represented by a matrix, which is often referred to as the information flow matrix (Davison and Chang, 1990).

A special case of structurally constrained controllers is when the local controller of each subsystem operates independently of the other subsystems, i.e., there is no di-

* This work has been supported by the Natural Sciences and Engineering Research Council of Canada under grant RGPIN-262127-07.

Email addresses: lavaei@cds.caltech.edu (Javad Lavaei), aghdam@ece.concordia.ca (Amir G. Aghdam). rect influence of the output of each subsystem on the control signal of other subsystems. This case is of a particular interest in the control literature, and is usually referred to as the decentralized control problem (Wang and Davison, 1973; Siljak, 1991; Lavaei, Momeni, and Aghdam, 2008; Lavaei and Aghdam, 2007c). Each control component in a decentralized control system observes only the output of its corresponding subsystem to construct the input of that subsystem. The notion of a decentralized fixed mode (DFM) was introduced in Wang and Davison (1973) to characterize the modes of an interconnected system which are fixed with respect to any decentralized linear time-invariant (LTI) controller. Since a DFM may not be fixed with respect to a nonlinear and time-varying controller, the notion of a quotient fixed mode (QFM) was introduced in Gong and Aldeen (1997) to identify those modes that are fixed with respect to any type of decentralized control law (i.e., nonlinear and time-varying). Since this notion was merely defined for strictly proper systems, it was further developed in Lavaei, Sojoudi, and Aghdam (2007) to identify the unwanted modes of any general proper system. Various properties of decentralized controllers have been investigated thoroughly in the literature (Siljak, 1991).

More recently, the case when the local controllers of an interconnected system can partially communicate with each other is studied intensively in the literature. This problem is referred to as decentralized overlapping con- 
trol (Zecevic and Siljak, 2005; Stankovic, Stanojevic, Siljak, 2000; Siljak and Zecevic, 2005), and is motivated by the following practical issues:

(1) The subsystems of many interconnected systems (referred to as overlapping subsystems) share some states (Siljak and Zecevic, 2005; Iftar, 1993, 1991). In this case, it is often desired that the structure of the controller matches the overlapping structure of the system (Siljak and Zecevic, 2005).

(2) In some systems, there are limitations on the availability of the states. In this case, only a subset of the outputs of the system are available for constructing each control signal, and the controller need not be localized like the conventional decentralized control structure (Lavaei and Aghdam, 2008).

The control constraint in both cases discussed above is described by an information flow matrix which reflects the desired control structure. Decentralized overlapping control can, in fact, be envisaged as a general case of traditional decentralized control problem. Note that the terms structurally constrained controller and decentralized overlapping controller are interchangeably used in the literature (Lavaei and Aghdam, 2007a, 2006; Ebihara and Hagiwara, 2003). Analogously to the definition of a DFM, the notion of a decentralized overlapping fixed mode (DOFM) was introduced in Lavaei and Aghdam (2008) to identify those modes of an interconnected system which are fixed with respect to LTI decentralized overlapping controllers. A procedure was then proposed to place the non-DOFMs freely in the complex plane. The question arises: Is there any non-LTI decentralized overlapping controller to shift a DOFM? It is also desired to employ the existing non-LTI decentralized control design techniques such as finite-dimensional linear timevarying (LTV), sampled-data, generalized sampled-data hold functions (GSHF), etc. to obtain a stabilizing decentralized overlapping controller.

This paper aims to address the above-mentioned questions. For this purpose, the mapping between the decentralized overlapping control and the decentralized control structures introduced in Lavaei and Aghdam (2008) is studied. This mapping brings about using the existing results on the traditional decentralized control design in order to solve the decentralized overlapping control design problem. Different types of decentralized overlapping control laws, namely sampled-data, GSHF, and finite-dimensional LTV are then investigated via this mapping. Moreover, the important problem of stabilizability of an interconnected system by means of a general (nonlinear and time-varying) decentralized overlapping controller is addressed via the new notion of a quotient overlapping fixed mode (QOFM). It is shown that any mode of the system is movable via a decentralized overlapping controller if and only if it is not a QOFM.

This paper is organized as follows. Some preliminary results, which are basically borrowed from Lavaei and Aghdam (2008), are presented in Section 2. The stabilizability with respect to decentralized overlapping controllers is studied in Section 3. The notion of analogousness is thoroughly investigated in Section 4 for several types of controllers, followed by two numerical examples in Section 5. Finally, some concluding remarks are given in Section 6.

\section{Preliminaries}

Consider a $\nu$-channel system $\mathcal{S}$ with the following statespace representation:

$$
\begin{aligned}
\dot{x}(t) & =A x(t)+\sum_{i=1}^{\nu} B_{i} u_{i}(t) \\
y_{i}(t) & =C_{i} x(t)+\sum_{j=1}^{\nu} D_{i j} u_{j}(t), i \in \bar{\nu}:=\{1,2, \ldots, \nu\}
\end{aligned}
$$

where $x(t) \in \Re^{n}$ is the state, and $u_{i}(t) \in \Re^{m_{i}}$ and $y_{i}(t) \in$ $\Re^{r_{i}}, i \in \bar{\nu}$, are the input and the output of the $i$-th channel of the system. Define now:

$$
\begin{aligned}
& u(t):=\left[\begin{array}{llll}
u_{1}(t)^{T} & u_{2}(t)^{T} & \cdots & u_{\nu}(t)^{T}
\end{array}\right]^{T}, \\
& y(t):=\left[\begin{array}{llll}
y_{1}(t)^{T} & y_{2}(t)^{T} & \cdots & y_{\nu}(t)^{T}
\end{array}\right]^{T}, \\
& B:=\left[\begin{array}{llll}
B_{1} & B_{2} & \cdots & B_{\nu}
\end{array}\right], \\
& C:=\left[\begin{array}{llll}
C_{1}^{T} & C_{2}^{T} & \cdots & C_{\nu}^{T}
\end{array}\right]^{T}, \\
& D:=\left[\begin{array}{ccc}
D_{11} & \cdots & D_{1 \nu} \\
\vdots & \ddots & \vdots \\
D_{\nu 1} & \cdots & D_{\nu \nu}
\end{array}\right] \text {, } \\
& m:=\sum_{i=1}^{\nu} m_{i}, \quad r:=\sum_{i=1}^{\nu} r_{i}
\end{aligned}
$$

It is desired to stabilize the system $\mathcal{S}$ by using a structurally constrained controller. The structure of this controller is determined by either of the matrices introduced below.

\section{Definition 1}

- Information flow matrix $\mathcal{K}$ is a matrix whose $(i, j)$ entry, $i, j \in \bar{\nu}$, is equal to 1 if the output $y_{j}(t)$ could contribute to the construction of the input $u_{i}(t)$ and is zero otherwise.

- Control interaction structure matrix $\mathbf{K}$ is a matrix whose $(i, j)$ block entry, $i, j \in \bar{\nu}$, is a $m_{i} \times r_{j}$ matrix denoted by the symbol $k_{i j}$ if the output $y_{j}(t)$ could contribute to the construction of the input $u_{i}(t)$ and is a $m_{i} \times r_{j}$ zero matrix otherwise. 
The information flow matrix corresponding to any system is enclosed in parentheses throughout the paper, if necessary. For instance, $\mathcal{S}(\mathcal{K})$ indicates that the structure of the controller to be designed for the system $\mathcal{S}$ is to comply with the information flow matrix $\mathcal{K}$.

It is noteworthy that $k_{i j}$ in Definition 1 represents the control channel which transforms the output $y_{j}(t)$ to the input $u_{i}(t)$. Note also that the interaction structure matrix $\mathbf{K}$ not only conveys the information of the matrix $\mathcal{K}$, but also labels the control components.

In order to present the main results of this work, some important concepts will be introduced first. Since $\mathbf{K}$ is not block-diagonal in general, it is preferable to expand it to a block-diagonal form. For this purpose, one can only perform the following operations:

- Introduce new block rows and block columns to the matrix $\mathbf{K}$.

- Swap the existing block rows of $\mathbf{K}$.

- Swap the existing block columns of $\mathbf{K}$.

However, this should be carried out in such a way that both of the following criteria hold:

- The resultant block-diagonal matrix has the same nonzero block entries as $\mathbf{K}$.

- If two nonzero block entries in the resultant matrix lie in the same block row (column), they are in the same block row (column) in the matrix $\mathbf{K}$ as well.

The informal expansion method described above is spelled out in detail in Lavaei and Aghdam (2008) (Procedures 1, 2 and 3). Denote all matrices obtained using this expansion with $\mathbf{K}_{1}, \mathbf{K}_{2}, \ldots, \mathbf{K}_{l}$ and, with no loss of generality, assume that $\mathbf{K}_{1}$ has the same number of columns as $\mathbf{K}$. For any $\mu \in \bar{l}:=\{1,2, \ldots, l\}$, let $m^{\mu} \times r^{\mu}$ denote the dimension of $\mathbf{K}_{\mu}$. Moreover, denote the size of the $i$-th diagonal block of $\mathbf{K}_{\mu}$ with $m_{i}^{\mu} \times r_{i}^{\mu}$.

Lemma 1 (Lavaei and Aghdam (2008)) There exist constant matrices $\Phi_{\mu}$ and $\bar{\Phi}_{\mu}$ satisfying the relation:

$$
\mathbf{K}=\Phi_{\mu} \mathbf{K}_{\mu} \bar{\Phi}_{\mu}
$$

for any $\mu \in \bar{l}$.

A simple algorithm is also proposed in Lavaei and Aghdam (2008) to obtain the transformation matrices introduced in Lemma 1.

Definition 2 Define $\mathcal{S}_{\mu}, \mu \in \bar{l}$, as an interconnected system with the following state-space representation:

$$
\begin{aligned}
& \dot{\mathbf{x}}_{\mu}(t)=A \mathbf{x}_{\mu}(t)+\mathbf{B}^{\mu} \mathbf{u}_{\mu}(t) \\
& \mathbf{y}_{\mu}(t)=\mathbf{C}^{\mu} \mathbf{x}_{\mu}(t)+\mathbf{D}^{\mu} \mathbf{u}_{\mu}(t)
\end{aligned}
$$

where the system parameters are related to the state-space matrices of the system $\mathcal{S}$ given by (1), as shown below:

$$
\mathbf{B}^{\mu}=B \Phi_{\mu}, \quad \mathbf{C}^{\mu}=\bar{\Phi}_{\mu} C, \quad \mathbf{D}^{\mu}=\bar{\Phi}_{\mu} D \Phi_{\mu}
$$

$\mathbf{u}_{\mu}(t) \in \Re^{m^{\mu}}$ and $\mathbf{y}_{\mu}(t) \in \Re^{r^{\mu}}$ are the input and the output of $\mathcal{S}_{\mu}$, respectively, and $\mathbf{x}_{\mu}(0)=x(0)$. Define also the information flow matrix $\mathcal{K}_{\mu}$ associated with the system $\mathcal{S}_{\mu}$ as a matrix obtained from $\mathbf{K}_{\mu}$ by replacing each of its nonzero entries (namely $k_{i j}$ ) with the scalar 1 , and each of its block zeros with the scalar 0.

Partition now the matrices $\mathbf{B}^{\mu}, \mathbf{C}^{\mu}$ and $\mathbf{D}^{\mu}, \mu \in \bar{l}$, as follows:

$$
\begin{aligned}
\mathbf{B}^{\mu} & =\left[\begin{array}{cccc}
\mathbf{B}_{1}^{\mu} & \mathbf{B}_{2}^{\mu} & \cdots & \mathbf{B}_{\nu_{\mu}}^{\mu}
\end{array}\right], \\
\mathbf{C}^{\mu} & =\left[\begin{array}{c}
\mathbf{C}_{1}^{\mu} \\
\mathbf{C}_{2}^{\mu} \\
\vdots \\
\mathbf{C}_{\nu_{\mu}}^{\mu}
\end{array}\right], \quad \mathbf{D}^{\mu}=\left[\begin{array}{ccc}
\mathbf{D}_{1,1}^{\mu} & \cdots & \mathbf{D}_{1, \nu_{\mu}}^{\mu} \\
\vdots & \ddots & \vdots \\
\mathbf{D}_{\nu_{\mu}, 1}^{\mu} & \cdots & \mathbf{D}_{\nu_{\mu}, \nu_{\mu}}^{\mu}
\end{array}\right]
\end{aligned}
$$

where $\nu_{\mu}$ denotes the number of block-diagonal entries of $\mathbf{K}_{\mu}$, and:

$$
\mathbf{B}_{i}^{\mu} \in \Re^{m_{i}^{\mu}}, \quad \mathbf{C}_{i}^{\mu} \in \Re^{r_{i}^{\mu}}, \quad \mathbf{D}_{i, j}^{\mu} \in \Re^{r_{i}^{\mu} \times m_{j}^{\mu}}
$$

for any $i, j \in\left\{1,2, \ldots, \nu_{\mu}\right\}$.

Definition 3 (Lavaei and Aghdam (2008)) Consider two arbitrary systems $\mathcal{S}_{d_{1}}$ and $\mathcal{S}_{d_{2}}$ associated with the information flow matrices $\mathcal{K}_{d_{1}}$ and $\mathcal{K}_{d_{2}}$, where $\mathcal{S}_{d_{1}}$ and $\mathcal{S}_{d_{2}}$ are of the same order and have the same initial state. Let $\mathbf{M}$ denote a given set of controllers. The systems $\mathcal{S}_{d_{1}}\left(\mathcal{K}_{d_{1}}\right)$ and $\mathcal{S}_{d_{2}}\left(\mathcal{K}_{d_{2}}\right)$ are called analogous with respect to (w.r.t.) $\mathbf{M}$ if for any controller $K_{d_{1}}$ in $\mathbf{M}$ complying with the information flow matrix $\mathcal{K}_{d_{1}}$, there also exists a controller $K_{d_{2}}$ in $\mathbf{M}$ complying with the information flow matrix $\mathcal{K}_{d_{2}}$ (and vice versa), such that the state of the system $\mathcal{S}_{d_{1}}$ under the controller $K_{d_{1}}$ is equivalent to the state of $\mathcal{S}_{d_{2}}$ under $K_{d_{2}}$.

One of the main objectives of this work is to prove that the systems $\mathcal{S}(\mathcal{K}), \mathcal{S}_{1}\left(\mathcal{K}_{1}\right), \mathcal{S}_{2}\left(\mathcal{K}_{2}\right), \ldots, \mathcal{S}_{l}\left(\mathcal{K}_{l}\right)$ are analogous w.r.t. several classes of controllers, including finitedimensional LTI and LTV controllers. The significance of this result will now be spelled out.

Consider a set of controllers denoted by $\mathbf{M}$. Assume that $\mathcal{S}(\mathcal{K})$ and $\mathcal{S}_{\mu}\left(\mathcal{K}_{\mu}\right), \mu \in \bar{l}$, are analogous w.r.t. M. In order to design a controller belonging to $\mathbf{M}$ for the system $\mathcal{S}$ w.r.t. the information flow structure $\mathcal{K}$ to achieve any design objectives, one can equivalently design a controller belonging to $\mathbf{M}$ for the system $\mathcal{S}_{\mu}$, w.r.t. the information flow structure $\mathcal{K}_{\mu}$, to attain the same objectives. The mapping between the components of $\mathbf{K}$ and 
$\mathbf{K}_{\mu}$ (derived from the equation (3)) can then be used to find the corresponding controller for the system $\mathcal{S}(\mathcal{K})$. The important advantage of this indirect design procedure is that the information flow structure $\mathcal{K}_{\mu}$ is blockdiagonal, and hence using the above mapping the problem is converted to the conventional decentralized control design framework.

\section{Decentralized overlapping stabilizability}

It is desired to find out under what conditions the system $\mathcal{S}(\mathcal{K})$ is stabilizable. To this end, the following notion is introduced.

Definition $4 A$ mode $\lambda \in s p(A)$ is a quotient overlapping fixed mode (QOFM) of the system $\mathcal{S}$ w.r.t. the information flow matrix $\mathcal{K}$, if it cannot be eliminated by using any type of controller whose structure complies with $\mathcal{K}$.

It is desired now to characterize the QOFMs of the system $\mathcal{S}(\mathcal{K})$. The next two lemmas are essential for this purpose.

Lemma 2 The sets of the QFMs of the systems $\mathcal{S}_{\mu}\left(\mathcal{K}_{\mu}\right), \mu \in \bar{l}$, are all identical.

Proof: The proof can be deduced from the following hierarchy of arguments:

- The systems $\mathcal{S}_{1}\left(\mathcal{K}_{1}\right), \ldots, \mathcal{S}_{l}\left(\mathcal{K}_{l}\right)$ all have the same $A$ matrix, and hence the same modes.

- It is shown in Gong and Aldeen (1997) and Anderson and Moore (1981) that all of the non-QFMs of any system can be eliminated by using a proper finitedimensional LTV controller.

- As will be proved later in Theorem 4, the systems $\mathcal{S}_{1}\left(\mathcal{K}_{1}\right), \ldots, \mathcal{S}_{l}\left(\mathcal{K}_{l}\right)$ are all analogous w.r.t. the set of finite-dimensional LTV controllers.

Lemma 3 The systems $\mathcal{S}(\mathcal{K})$ and $\mathcal{S}_{1}\left(\mathcal{K}_{1}\right)$ are analogous w.r.t. the class of all (nonlinear and time-varying) controllers.

Proof: The system $\mathcal{S}_{1}$ is obtained from $\mathcal{S}$ by introducing some redundant outputs and reordering them with the aim of converting the structure $\mathcal{K}$ to $\mathcal{K}_{1}$. The proof follows by noting that this system expansion does not change the internal behavior of the system.

Note that the systems $\mathcal{S}(\mathcal{K})$ and $\mathcal{S}_{\mu}\left(\mathcal{K}_{\mu}\right)$, are not necessarily analogous w.r.t. the set of nonlinear controllers if $\mu \neq 1$. This results from the fact that the proposed control structure conversion does not preserve the superposition property, in general.

The following theorem captures an elegant property of the QOFMs of $\mathcal{S}(\mathcal{K})$.
Theorem 1 The QOFMs of the system $\mathcal{S}(\mathcal{K})$ are the same as the QFMs of the system $\mathcal{S}_{\mu}\left(\mathcal{K}_{\mu}\right), \forall \mu \in \bar{l}$. Moreover, the system $\mathcal{S}(\mathcal{K})$ is stabilizable if and only if it does not have any unstable QOFM.

Proof: It can be concluded from Lemma 3 that the QOFMs of the system $\mathcal{S}(\mathcal{K})$ are the same as the QFMs of the system $\mathcal{S}_{1}\left(\mathcal{K}_{1}\right)$. On the other hand, the QFMs of $\mathcal{S}_{1}\left(\mathcal{K}_{1}\right)$ are identical to the QFMs of $\mathcal{S}_{\mu}\left(\mathcal{K}_{\mu}\right), \forall \mu \in \bar{l}$, as asserted in Lemma 2. These two observations together complete the first part of the proof. The second statement of the theorem follows directly from the definition of analogousness and the celebrated result which states that a system with no unstable QFMs can be stabilized using a proper structurally constrained controller.

\section{Decentralized overlapping controller design}

Assume that the system $\mathcal{S}(\mathcal{K})$ has no unstable QOFM. The question arises as to how to design a stabilizing controller of a desired form which satisfies any prescribed specifications. The raised question is answered in Lavaei and Aghdam (2008) for designing a LTI overlapping controller. For the case of non-LTI controllers, however, the answer would require a meticulous study, which will be carried out below.

\subsection{Generalized sampled-data hold function}

Periodic control design using generalized sampled-data hold function (GSHF) and its advantages have been studied intensively in the literature (Kabamba, 1987; Rossi and Miller, 1999; Lavaei and Aghdam, 2007a,b). Assume that it is desired to obtain a GSHF for the system $\mathcal{S}$ complying with the information flow structure $\mathcal{K}$ in order to achieve certain design objectives. Let this GSHF be denoted by $F(t)$. Hence, the resultant control signal can be formulated as follows:

$u(t)=F(t) y[\kappa], \quad \kappa h \leq t<(\kappa+1) h, \quad \kappa \geq 0$

where $h$ represents the sampling period. Note that the discrete argument corresponding to the samples of any signal is enclosed in brackets (e.g., $y[\kappa]:=y(\kappa h))$. In this subsection, assume that $D$ is a zero matrix.

Theorem 2 The systems $\mathcal{S}(\mathcal{K}), \mathcal{S}_{1}\left(\mathcal{K}_{1}\right), \ldots, \mathcal{S}_{l}\left(\mathcal{K}_{l}\right)$ are analogous w.r.t. the set of all GSHF-type controllers.

Proof: To prove the theorem, it suffices to show that $\mathcal{S}(\mathcal{K})$ and $\mathcal{S}_{\mu}\left(\mathcal{K}_{\mu}\right)$ are analogous w.r.t. all GSHF-type controllers, for any $\mu \in \bar{l}$. Consider a GSHF $F(t)$ which complies with the information flow structure $\mathcal{K}$. Utilize the proper transformation on $F(t)$ to obtain the equivalent hold function $F_{\mu}(t)$ for the system $\mathcal{S}_{\mu}\left(\mathcal{K}_{\mu}\right)$. Note that $F_{\mu}(t)$ can be attained using the mapping between the components of $\mathbf{K}$ and $\mathbf{K}_{\mu}$. Since $F(t)$ and $F_{\mu}(t)$ 
comply with the information flow matrices $\mathcal{K}$ and $\mathcal{K}_{\mu}$, respectively, it is straightforward to show that $F(t)=$ $\Phi_{\mu} F_{\mu}(t) \bar{\Phi}_{\mu}$. On the other hand, it follows from (8) that:

$$
\dot{x}(t)=A x(t)+B F(t) C x[\kappa]
$$

for all $t \in[\kappa h,(\kappa+1) h), \kappa \geq 0$, and consequently:

$$
\begin{aligned}
\dot{\mathbf{x}}_{\mu}(t) & =A \mathbf{x}_{\mu}(t)+\mathbf{B}^{\mu} F_{\mu}(t) \mathbf{C}^{\mu} \mathbf{x}_{\mu}[\kappa] \\
& =A \mathbf{x}_{\mu}(t)+B \Phi_{\mu} F_{\mu}(t) \bar{\Phi}_{\mu} C \mathbf{x}_{\mu}[\kappa] \\
& =A \mathbf{x}_{\mu}(t)+B F(t) C \mathbf{x}_{\mu}[\kappa]
\end{aligned}
$$

in the above-mentioned time-interval. The equations (9) and (10), and the equality $x(0)=\mathbf{x}_{\mu}(0)$ result in the relation $x(t)=\mathbf{x}_{\mu}(t)$ for all $t \geq 0$. Conversely, for any GSHF $F_{\mu}(t)$ complying with the information flow matrix $\mathcal{K}_{\mu}$, it is straightforward to show that the state of the system $\mathcal{S}$ under the GSHF $F(t)=\Phi_{\mu} F_{\mu}(t) \bar{\Phi}_{\mu}$ is identical to that of the system $\mathcal{S}_{\mu}$ under $F_{\mu}(t)$.

Theorem 2 states that the problem of designing a GSHF for the system $\mathcal{S}(\mathcal{K})$ can be formulated as the problem of designing a GSHF for the system $\mathcal{S}_{\mu}\left(\mathcal{K}_{\mu}\right)$ for any $\mu \in \bar{l}$. However, due to the decentralized control structure of $\mathcal{S}_{\mu}\left(\mathcal{K}_{\mu}\right), \mu \in \bar{l}$, the corresponding GSHF design can be accomplished by using the existing methods (Lavaei and Aghdam, 2007a,b; Aghdam, 2006).

\subsection{Sampled-data controller}

A typical sampled-data controller consists of a sampler, a zero-order hold $(\mathrm{ZOH})$ and a discrete-time controller (Chen and Francis, 1995). It is desired in this subsection to present a method for designing a sampled-data controller for the system $\mathcal{S}$, whose structure complies with a given information flow matrix $\mathcal{K}$. The term linear shift-invariant (LSI) controller will henceforth refer to a sampled-data controller which is LTI w.r.t. the discretetime equivalent model of the system $\mathcal{S}$. It is worth mentioning that a LSI controller is, in fact, LTV w.r.t. the original continuous-time system.

Theorem 3 The systems $\mathcal{S}(\mathcal{K}), \mathcal{S}_{1}\left(\mathcal{K}_{1}\right), \ldots, \mathcal{S}_{l}\left(\mathcal{K}_{l}\right)$ are all analogous w.r.t. the set of all LSI controllers.

Proof: Denote the sampling period with $h$, and the discrete-time equivalent models of the systems $\mathcal{S}, \mathcal{S}_{1}, \ldots, \mathcal{S}_{l}$ with $\overline{\mathcal{S}}, \overline{\mathcal{S}}_{1}, \ldots, \overline{\mathcal{S}}_{l}$, respectively. Assume that the system $\overline{\mathcal{S}}$ is represented by:

$$
\begin{aligned}
x[\kappa+1] & =\bar{A} x[\kappa]+\bar{B} u[\kappa] \\
y[\kappa] & =C x[\kappa]+D u[\kappa]
\end{aligned}
$$

Similarly, let the system $\overline{\mathcal{S}}_{\mu}$ be represented by:

$$
\begin{aligned}
\mathbf{x}_{\mu}[\kappa+1] & =\bar{A} \mathbf{x}_{\mu}[\kappa]+\overline{\mathbf{B}}^{\mu} \mathbf{u}_{\mu}[\kappa] \\
\mathbf{y}_{\mu}[\kappa] & =\mathbf{C}^{\mu} \mathbf{x}_{\mu}[\kappa]+\mathbf{D}^{\mu} \mathbf{u}_{\mu}[\kappa], \quad \mu \in \bar{l}
\end{aligned}
$$

It can be easily verified that:

$$
\overline{\mathbf{B}}^{\mu}=\int_{0}^{h} e^{\tau A} \mathbf{B}^{\mu} d \tau=\int_{0}^{h} e^{\tau A} B d \tau \times \Phi_{\mu}=\bar{B} \Phi_{\mu}
$$

It results from (11), (12), and (13) that the state-space matrices of $\overline{\mathcal{S}}$ are related to those of $\overline{\mathcal{S}}_{\mu}$, exactly in the same way the state-space matrices of $\mathcal{S}$ and $\mathcal{S}_{\mu}$ are related. Hence, the systems $\overline{\mathcal{S}}$ and $\overline{\mathcal{S}}_{\mu}$ are analogous w.r.t. the LSI controllers. Consider now a LSI controller with the transfer function matrix $\bar{K}(z)$ for the system $\overline{\mathcal{S}}(\mathcal{K})$. Construct a LSI controller with the transfer function matrix $\bar{K}_{\mu}(z)$ for the system $\overline{\mathcal{S}}_{\mu}\left(\mathcal{K}_{\mu}\right)$, such that it corresponds to the controller $\bar{K}(z)$ for $\overline{\mathcal{S}}(\mathcal{K})$. This controller can be obtained from the mapping between the components of $\mathbf{K}$ and $\mathbf{K}_{\mu}$. It is straightforward to show that $\bar{K}(z)=\Phi_{\mu} \bar{K}_{\mu}(z) \bar{\Phi}_{\mu}$. Applying the controller $\bar{K}(z)$ to the system $\overline{\mathcal{S}}$ and the controller $\bar{K}_{\mu}(z)$ to $\overline{\mathcal{S}}_{\mu}$, one can conclude that $x[\kappa]=\mathbf{x}_{\mu}[\kappa]$ and $u[\kappa]=\Phi_{\mu} \mathbf{u}_{\mu}[\kappa]$ for any $\kappa \geq 0$. Therefore:

$$
\begin{aligned}
x(t) & =e^{(t-\kappa h) A} x[\kappa]+\int_{\kappa h}^{t} e^{(\tau-\kappa h) A} B u[\kappa] d \tau \\
& =e^{(t-\kappa h) A} \mathbf{x}_{\mu}[\kappa]+\int_{\kappa h}^{t} e^{(\tau-\kappa h) A} B \Phi_{\mu} \mathbf{u}_{\mu}[\kappa] d \tau \\
& =e^{(t-\kappa h) A} \mathbf{x}_{\mu}[\kappa]+\int_{\kappa h}^{t} e^{(\tau-\kappa h) A} \mathbf{B}^{\mu} \mathbf{u}_{\mu}[\kappa] d \tau \\
& =\mathbf{x}_{\mu}(t)
\end{aligned}
$$

for any $t \in[\kappa h,(\kappa+1) h), k \geq 0$. Similarly, it can be easily verified that given any controller $\bar{K}_{\mu}(z)$ for the system $\overline{\mathcal{S}}_{\mu}(\mathcal{K})$, the controller $\bar{K}(z):=\Phi_{\mu} \bar{K}_{\mu}(z) \bar{\Phi}_{\mu}$ corresponds to the information flow matrix $\mathcal{K}$, and that the state of the system $\mathcal{S}$ under the controller $\bar{K}(z)$ is the same as that of $\mathcal{S}_{\mu}$ under $\bar{K}_{\mu}(z)$.

Note that finding a sampled-data decentralized control law to achieve any desired design objectives has been investigated in the literature; e.g, see Lavaei et al. (2006).

\subsection{Finite-dimensional linear time-varying controller}

It is well-known that finite-dimensional linear timevarying (LTV) controllers are superior to their LTI counterparts in many control applications. It is desired in this subsection to present a procedure for designing a finite-dimensional LTV controller complying with the information flow matrix $\mathcal{K}$, for the system $\mathcal{S}$. The term "finite-dimensional LTV controller" refers in this paper to a control law which can be represented as:

$$
\begin{aligned}
\dot{\tilde{z}}(t) & =\tilde{A}(t) \tilde{z}(t)+\tilde{B}(t) \tilde{u}(t) \\
\tilde{y}(t) & =\tilde{C}(t) \tilde{z}(t)+\tilde{D}(t) \tilde{u}(t)
\end{aligned}
$$


Theorem 4 The systems $\mathcal{S}(\mathcal{K}), \mathcal{S}_{1}\left(\mathcal{K}_{1}\right), \ldots, \mathcal{S}_{l}\left(\mathcal{K}_{l}\right)$ are analogous w.r.t. the set of all finite-dimensional LTV controllers.

Rather than presenting a formal proof here, the following insightful discussion is provided to clarify the main idea of the proof and the reason why this structure conversion is introduced.

One can easily verify that $\mathbf{B}^{\mu}, \mu \in \bar{l}$, is extracted from $B$ by rearranging its columns and repeating some of them. Analogously, $\mathbf{C}^{\mu}$ is extracted from $C$ by rearranging its rows and repeating some of them. The reason for these repetitions and rearrangements is described below:

1. Repetition of the rows of $C^{\mu}$ reflects the fact that some of the outputs of $\mathcal{S}$ are duplicated to construct the system $\mathcal{S}_{\mu}$. To justify the necessity of this duplication, assume that one output of the system $\mathcal{S}$ directly influences two distinct control inputs, resulting in a non-diagonal information flow structure. Now, if this output of the system is duplicated and directed to one of the two control agents mentioned above, while its duplicate goes to the other control agent, the resultant controller from the input's point of view would be decentralized. However, both closed-loop systems (the original one and the one obtained following the above procedure) are basically the same.

2. Regarding the repetition in the columns of $\mathbf{B}^{\mu}$, assume that two outputs of the system go to one control agent. Since the controller is linear, one can split it into two sub-controllers such that each of the two outputs goes to one of these sub-control agents. In addition, the output of the original control agent is the sum of the outputs of these two sub-control agents. Again, the functionality of the control system is unaltered, while its structure is converted to a decentralized form.

3. The rearrangement of the rows and the columns of $C$ and $B$ in constructing $C^{\mu}$ and $B^{\mu}$ means that the inputs and outputs of $\mathcal{S}$ are reordered (i.e., the corresponding indices are renumbered), which again would not affect the functionality of the control system.

Taking the aforementioned issues into consideration, the system $\mathcal{S}_{\mu}$ is indeed constructed from $\mathcal{S}$ in such a way that the control structure $\mathcal{K}$ is converted to the decentralized structure $\mathcal{K}_{\mu}$, without affecting the functionality of the overall system.

Remark 1 It is shown in Anderson and Moore (1981) that a system is decentrally stabilizable if and only if there exists a decentralized finite-dimensional LTV controller to stabilize it. Applying this result to the system $\mathcal{S}_{\mu}\left(\mathcal{K}_{\mu}\right)$ and exploiting Theorem 4 , one can conclude that $\mathcal{S}(\mathcal{K})$ is stabilizable if and only if there exists a finite-dimensional LTV controller which complies with $\mathbf{K}$ and stabilizes the system. If such a controller exists, it can be designed by employing the concept of analogousness.

\section{$5 \quad$ Numerical examples}

Example 1: Let $\mathcal{S}$ be a four-SISO-channel system with the following Jordan matrices:

$$
A=\left[\begin{array}{cccc}
1 & 0 & 0 & 0 \\
0 & -1 & 0 & 0 \\
0 & 0 & 3 & 0 \\
0 & 0 & 0 & -3
\end{array}\right], \quad B=\left[\begin{array}{cccc}
0 & 0 & 2 & -1 \\
-1 & 0 & 0 & 0 \\
4 & 3 & 2 & 1 \\
3 & 0 & 0 & 0
\end{array}\right]
$$

and

$$
C=\left[\begin{array}{cccc}
0 & 0 & 0 & -1 \\
2 & 3 & -1 & 2 \\
0 & 3 & 0 & 0 \\
0 & -2 & -3 & -4
\end{array}\right], \quad D=\left[\begin{array}{cccc}
1 & 0 & 0 & 0 \\
5 & 3 & 2 & 8 \\
3 & 0 & 0 & 1 \\
1 & -1 & 0 & 1
\end{array}\right]
$$

Assume that the desired control interaction structure is:

$$
\mathbf{K}=\left[\begin{array}{cccc}
k_{11} & 0 & 0 & 0 \\
0 & k_{22} & k_{23} & 0 \\
0 & 0 & k_{33} & k_{34} \\
k_{41} & k_{42} & k_{43} & 0
\end{array}\right]
$$

Using Procedures 1, 2, and 3 introduced in Lavaei and Aghdam (2008), one can construct the matrix $\mathbf{K}_{2}$ as follows:

$$
\mathbf{K}_{2}=\left[\begin{array}{ccccc}
k_{22} & k_{23} & 0 & 0 & 0 \\
k_{42} & k_{43} & 0 & 0 & 0 \\
0 & 0 & k_{33} & k_{34} & 0 \\
0 & 0 & 0 & 0 & k_{11} \\
0 & 0 & 0 & 0 & k_{41}
\end{array}\right]
$$

(note that this matrix is denoted by $\mathbf{K}_{2}$ instead of $\mathbf{K}_{1}$, because the notation $\mathbf{K}_{1}$ has been reserved for a particular matrix transformation). As a result, following the method given in Lavaei and Aghdam (2008), the matrices $\Phi_{2}$ and $\bar{\Phi}_{2}$ satisfying the relation $\mathbf{K}=\Phi_{2} \mathbf{K}_{2} \bar{\Phi}_{2}$ are obtained as follows:

$$
\Phi_{2}=\left[\begin{array}{lllll}
0 & 0 & 0 & 1 & 0 \\
1 & 0 & 0 & 0 & 0 \\
0 & 0 & 1 & 0 & 0 \\
0 & 1 & 0 & 0 & 1
\end{array}\right], \quad \bar{\Phi}_{2}=\left[\begin{array}{llll}
0 & 1 & 0 & 0 \\
0 & 0 & 1 & 0 \\
0 & 0 & 1 & 0 \\
0 & 0 & 0 & 1 \\
1 & 0 & 0 & 0
\end{array}\right]
$$

Consequently, the system $\mathcal{S}_{2}$ can be straightforwardly formed according to Definition 2. It results from Lavaei, 
Sojoudi, and Aghdam (2007) that $\lambda=-1$ is a QFM of the system $\mathcal{S}_{2}\left(\mathcal{K}_{2}\right)$, which implies that this mode is a QOFM of the system $\mathcal{S}(\mathcal{K})$ (in light of Theorem 1).This means that there is no structurally constrained controller complying with $\mathcal{K}$ to displace this mode. However, since $\lambda=-1$ is a stable mode, there exists a LTV stabilizing structurally constrained controller for the system as stated earlier.

Example 2: Let the system $\mathcal{S}$ be represented by the following state-space matrices:

$$
\begin{aligned}
A & =\left[\begin{array}{cccccc}
-1.5 & -1 & -1 & -2 & -1 & -2.5 \\
-1.5 & -2 & 0 & 2 & 0 & 1.5 \\
0 & 0 & -3 & 0 & 0 & 0 \\
-1 & -1 & -1 & -3 & -1 & -1 \\
1.5 & 3 & 0 & -2 & 1 & -1.5 \\
-0.5 & 1 & 1 & 2 & 1 & 0.5
\end{array}\right], \\
B= & {\left[\begin{array}{ccccc}
0 & 2 & -0.5 & -0.5 \\
0 & 1 & 0.5 & -2.5 \\
0 & 1 & 0 & 0 \\
0 & 1 & 0 & -1 \\
1 & -1 & -0.5 & 2.5 \\
0 & 1 & 0.5 & 0.5
\end{array}\right] } \\
C= & {\left[\begin{array}{cccccc}
-1 & 0 & 1 & 0 & 0 & 1 \\
0 & 1 & 1 & 0 & 1 & 0 \\
1 & 0 & -4 & 0 & 0 & 1 \\
0 & -1 & -4 & 4 & 0 & 1
\end{array}\right] }
\end{aligned}
$$

and $D=0$. Assume that the desired control interaction structure is:

$$
\mathbf{K}=\left[\begin{array}{cccc}
0 & 0 & k_{13} & k_{14} \\
0 & k_{22} & k_{23} & k_{24} \\
0 & 0 & k_{33} & k_{34} \\
k_{41} & 0 & 0 & 0
\end{array}\right]
$$

Using Procedures 1, 2 and 3 introduced in Lavaei and Aghdam (2008), the block-diagonal matrix $\mathbf{K}_{1}$ can be obtained as:

$$
\mathbf{K}_{1}=\left[\begin{array}{cccc}
k_{13} & k_{14} & 0 & 0 \\
k_{23} & k_{24} & 0 & 0 \\
k_{33} & k_{34} & 0 & 0 \\
0 & 0 & k_{22} & 0 \\
0 & 0 & 0 & k_{41}
\end{array}\right]
$$

Hence, the matrices $\Phi_{1}$ and $\bar{\Phi}_{1}$ can be found using the method given in Lavaei and Aghdam (2008). Consequently, the system $\mathcal{S}_{1}$ can be straightforwardly formed. As pointed out in Lavaei and Aghdam (2008), the DOFMs of the system $\mathcal{S}(\mathcal{K})$ are identical to the DFMs of the system $\mathcal{S}_{1}\left(\mathcal{K}_{1}\right)$. It is easy to verify that $\mathcal{S}_{1}\left(\mathcal{K}_{1}\right)$ has a DFM at $\lambda=+1$; therefore, the system $\mathcal{S}$ has a DOFM at $\lambda=+1$ w.r.t. the information flow matrix $\mathcal{K}$. Thus, this system cannot be stabilized by means of a structurally constrained LTI controller complying with $\mathcal{K}$ (Lavaei and Aghdam, 2008).

On the other hand, it follows from the characterization of QFMs given in Gong and Aldeen (1997) that the system $\mathcal{S}_{1}\left(\mathcal{K}_{1}\right)$ has no QFM w.r.t. the information flow matrix $\mathcal{K}_{1}$. From the results of the present work, it is known that the QOFMs of the system $\mathcal{S}(\mathcal{K})$ are the same as the QFMs of the system $\mathcal{S}_{1}\left(\mathcal{K}_{1}\right)$. Thus, one can conclude that the system $\mathcal{S}$ has no QOFM w.r.t. the information flow matrix $\mathcal{K}$. This implies that the system $\mathcal{S}(\mathcal{K})$ can be stabilized by utilizing a proper non-LTI controller.

It is desired now to find a structurally constrained controller which stabilizes the system $\mathcal{S}(\mathcal{K})$. Due to the fact that the DFM of the system $\mathcal{S}_{1}$ w.r.t. $\mathcal{K}_{1}$ is not a $\mathrm{QFM}$, it can be eliminated by means of sampling, as pointed out in Lavaei and Aghdam (2006). To this end, choose the sampling period $h=3$, and find the discrete-time equivalent models of $\mathcal{S}$ and $\mathcal{S}_{1}$, denoted by $\overline{\mathcal{S}}$ and $\overline{\mathcal{S}}_{1}$, respectively. It can be easily verified that $\overline{\mathcal{S}}_{1}\left(\mathcal{K}_{1}\right)$ has no DFM, as expected. Moreover, the technique given in Anderson and Moore (1981) can be utilized to control the system from only one subsystem. More precisely, since the structural graph of the system $\overline{\mathcal{S}}_{1}$ is strongly connected, all the control components but $\bar{K}_{13}(z)$ can be generically chosen as:

$$
\begin{aligned}
& \bar{K}_{22}(z)=1, \quad \bar{K}_{24}(z)=2, \quad \bar{K}_{34}(z)=3 \\
& \bar{K}_{41}(z)=4, \quad \bar{K}_{14}(z)=\bar{K}_{23}(z)=\bar{K}_{33}(z)=0
\end{aligned}
$$

where $\bar{K}_{i j}(z)$ represents the discrete-time compensator for the system $\overline{\mathcal{S}}_{1}$, which along with a $\mathrm{ZOH}$ builds the corresponding control component $k_{i j}$. Let the closedloop system consisting of $\overline{\mathcal{S}}_{1}$ and all the controller components given in $(24)$ be denoted by $\overline{\mathcal{S}}_{1_{\mathrm{cl}}}$. This closedloop system is controllable and observable through/from the input and the output corresponding to the controller component $\bar{K}_{13}(z)$. Therefore, the controller $\bar{K}_{13}(z)$ can be designed for the system $\overline{\mathcal{S}}_{1_{\mathrm{cl}}}$ to not only stabilize the system but also place the modes at desired locations, by using the conventional pole-assignment techniques. Since $\mathcal{S}(\mathcal{K})$ and $\mathcal{S}_{1}\left(\mathcal{K}_{1}\right)$ are analogous w.r.t. the sampled-data controllers, the designed components for controlling the system $\mathcal{S}_{1}$ can be used for controlling the system $\mathcal{S}$ as well. Both closed-loop systems would perform identically. 


\section{Conclusions}

This work tackles the structurally constrained control design problem for interconnected systems. The conventional techniques for designing a decentralized controller with a desired type such as finite dimensional linear timevarying and generalized sampled-data hold functions are developed to handle the problem of designing an overlapping controller with any given information flow structure. The notion of a quotient overlapping fixed mode (QOFM) is also defined to investigate the stabilizability of the system via a general (nonlinear and time-varying) decentralized overlapping control law. It is shown that a mode of the system can be moved in the complex plane by means of a structurally constrained controller if and only if it is not a QOFM. Numerical examples illustrate the significance of the results.

\section{References}

Aghdam, A. G. (2006). Decentralized control design using piecewise constant hold functions. Proceedings of 2006 American Control Conference (pp. 207212).

Anderson, B. D. O., \& Moore, J. (1981). Timevarying feedback laws for decentralized control. IEEE Transactions on Automatic Control. 26(5), $1133-1139$.

Chen, T., \& Francis, B. (1995). Optimal sampled-data control systems, Springer,

Davison, E. J., \& Chang, T. N. (1990). Decentralized stabilization and pole assignment for general proper systems. IEEE Transactions on Automatic Control. $35(6), 652-664$.

Ebihara, Y., \& Hagiwara, T. (2003). Structured controller synthesis using LMI and alternating projection method. Proceedings of 42nd IEEE Conference on Decision and Control (pp. 5632-5637).

Iftar, A. (1991). Decentralized optimal control with overlapping decompositions. IEEE International Conference on Systems Engineering (pp. 299-302).

Iftar, A. (1993). Overlapping decentralized dynamic optimal control. International Journal of Control. 58(1), 187-209.

Gong, Z., \& Aldeen, M. (1997). Stabilization of decentralized control systems. Journal of Mathematical Systems, Estimation, and Control. 7(1), 1-16.

Kabamba, P. T. (1987). Control of linear systems using generalized sampled-data hold functions. IEEE Transactions on Automatic Control. 32(9), 772-783.

Lavaei, J., \& Aghdam, A. G. (2006). Elimination of fixed modes by means of high-performance constrained periodic control. Proceedings of 45 th IEEE Conference on Decision and Control (pp. 4441-4447).

Lavaei, J., \& Aghdam, A. G. (2007a). Optimal periodic feedback design for continuous-time LTI systems with constrained control structure. International Journal of Control. 80(2), 220-230.
Lavaei, J., \& Aghdam, A. G. (2007b). Simultaneous LQ control of a set of LTI systems using constrained generalized sampled-data hold functions. Automatica. 43(2), 274-280.

Lavaei, J., \& Aghdam, A. G. (2007c). High-performance decentralized control design for general interconnected systems with applications in cooperative control. International Journal of Control. 80(6), 935-951.

Lavaei, J., Momeni, A., \& Aghdam, A. G. (2008). Spacecraft formation control in deep space with reduced communication requirement. IEEE Transactions on Control Systems Technology. 16(2), 268-278.

Lavaei, J., Sojoudi, S., \& Aghdam, A. G. (2007). Stability criterion for general proper systems with constrained control structure. Proceedings of 2007 American Control Conference (pp. 1648-1653).

Lavaei, J., \& Aghdam, A. G. (2008). Control of continuous-time LTI systems by means of structurally constrained controllers. Automatica. 44(1), 141-148.

Rossi, M., \& Miller, D. E. (1999). Gain/phase margin improvement using static generalized sampled-data hold functions. Systems \& Control Letters. 37(3), $163-172$.

Šiljak, D. D. (1991). Decentralized control of complex systems, Cambridge: Academic Press.

Šiljak, D. D., \& Zecevic, A. I. (2005). Control of largescale systems: Beyond decentralized feedback. Annual Reviews in Control. 29(2), 169-179.

Stankovic, S. S., Stanojevic, M. J., \& Šiljak, D. D. (2000). Decentralized overlapping control of a platoon of vehicles. IEEE Transactions on Control Systems Technology. 8(5), 816-832.

Wang, S. H., \& Davison, E. J. (1973). On the stabilization of decentralized control systems. IEEE Transactions on Automatic Control. 18(5), 473-478.

Zecevic, A. I., \& Šiljak, D. D. (2005). A new approach to control design with overlapping information structure constraints. Automatica. 41(2), 265-272. 